\title{
ASPERGILLUS MEDIASTINITIS IN AN IMMUNOCOMPETENT CHILD
}

\author{
Vidur Bansal ${ }^{1}$, Anand Mishra ${ }^{2}$, gradlin roy $^{2}$, Vikram Halder $^{2}$, and parakritii gupta ${ }^{2}$ \\ ${ }^{1}$ PGIMER \\ ${ }^{2}$ Post Graduate Institute of Medical Education and Research
}

April 27, 2020

\begin{abstract}
Mediastinitis is a serious complication after cardiac surgery. While bacteria are the more common pathogens, fungal infections are rare. Post-operative Aspergillus mediastinitis is considered to be a catastrophic infection, affecting patients with specific predisposing factors undergoing cardiothoracic surgery. The patient outcome after aspergillus mediastinitis is extremely poor despite antifungal therapy and surgery. The diagnosis is usually delayed and relies on direct visualization and culture. Clinical features of post-operative Aspergillus mediastinitis could be minimal, underlining the necessity for a low index of suspicion in cases of culture-negative mediastinitis. Antifungal therapy including amphotericin B or voriconazole along with surgical debridement forms the mainstay of treatment.
\end{abstract}

\section{ASPERGILLUS MEDIASTINITIS IN AN IMMUNOCOMPETENT CHILD}

\section{AUTHORS}

Vidur Bansal ${ }^{1}$, Anand Kumar Mishra ${ }^{1}$, Parakritii Gupta ${ }^{2}$, Gradlin Roy ${ }^{1}$, Vikram Halder ${ }^{1}$

1. Department of Cardiothoracic and Vascular Surgery(CTVS), Advanced cardiac Centre(ACC), PGIMER, Chandigarh, India

2. Department of Medical Microbiology, PGIMER, Chandigarh, India

CORRESPONDING AUTHOR

Dr. Vidur Bansal, Department of CTVS, ACC, PGIMER, Chandigarh, India

RUNNING HEAD - Aspergillus mediastinitis

KEYWORDS - Aspergillus; immunocompetent; antifungal

\section{ABSTRACT}

Mediastinitis is a serious complication after cardiac surgery. While bacteria are the more common pathogens, fungal infections are rare. Post-operative Aspergillus mediastinitis is considered to be a catastrophic infection, affecting patients with specific predisposing factors undergoing cardiothoracic surgery. The patient outcome after aspergillus mediastinitis is extremely poor despite antifungal therapy and surgery. The diagnosis is usually delayed and relies on direct visualization and culture. Clinical features of post-operative Aspergillus 
mediastinitis could be minimal, underlining the necessity for a low index of suspicion in cases of culturenegative mediastinitis. Antifungal therapy including amphotericin B or voriconazole along with surgical debridement forms the mainstay of treatment.

\section{TEXT}

Post-operative Aspergillus mediastinitis is regarded to be a devastating infection, usually affecting patients undergoing cardiothoracic surgery with specific predisposing factors. ${ }^{1}$ We describe the case of an immunocompetent 2-month-old child with obstructed Supracardiac Total Anomalous Pulmonary Venous Circulation(TAPVC) and severe Pulmonary Artery Hypertension(PAH), who underwent TAPVC repair via median sternotomy. Primary closure of the sternum was not possible due to significant myocardial oedema, necessitating a polyvinylchloride(PVC) patch to be sutured to the skin. The child was put on broad spectrum antibiotics from the outset and liposomal amphotericin B was started on POD 8. But the child died as a result of septic shock and multiple organ failure on POD-19. Pericardial and sternal tissues were positive for hyphae under direct visualization, compatible with Aspergillus species. Cultures were positive for several colonies of Aspergillus flavus, no bacteria were isolated. A comprehensive search for possible environmental contamination did not reveal any source in the operating room or in the ICU. In this patient, the maintenance of chest opening for over 7 days appeared to be the most probable entry site of infection.

Post-surgical mediastinitis is defined as an infection involving the structures between the sternum and the esophagus, occurring mainly after cardiothoracic surgery. ${ }^{2}$ Optimal treatment relies on surgical debridement and antifungal therapy Antifungal treatment should include amphotericin B or voriconazole. ${ }^{3}$

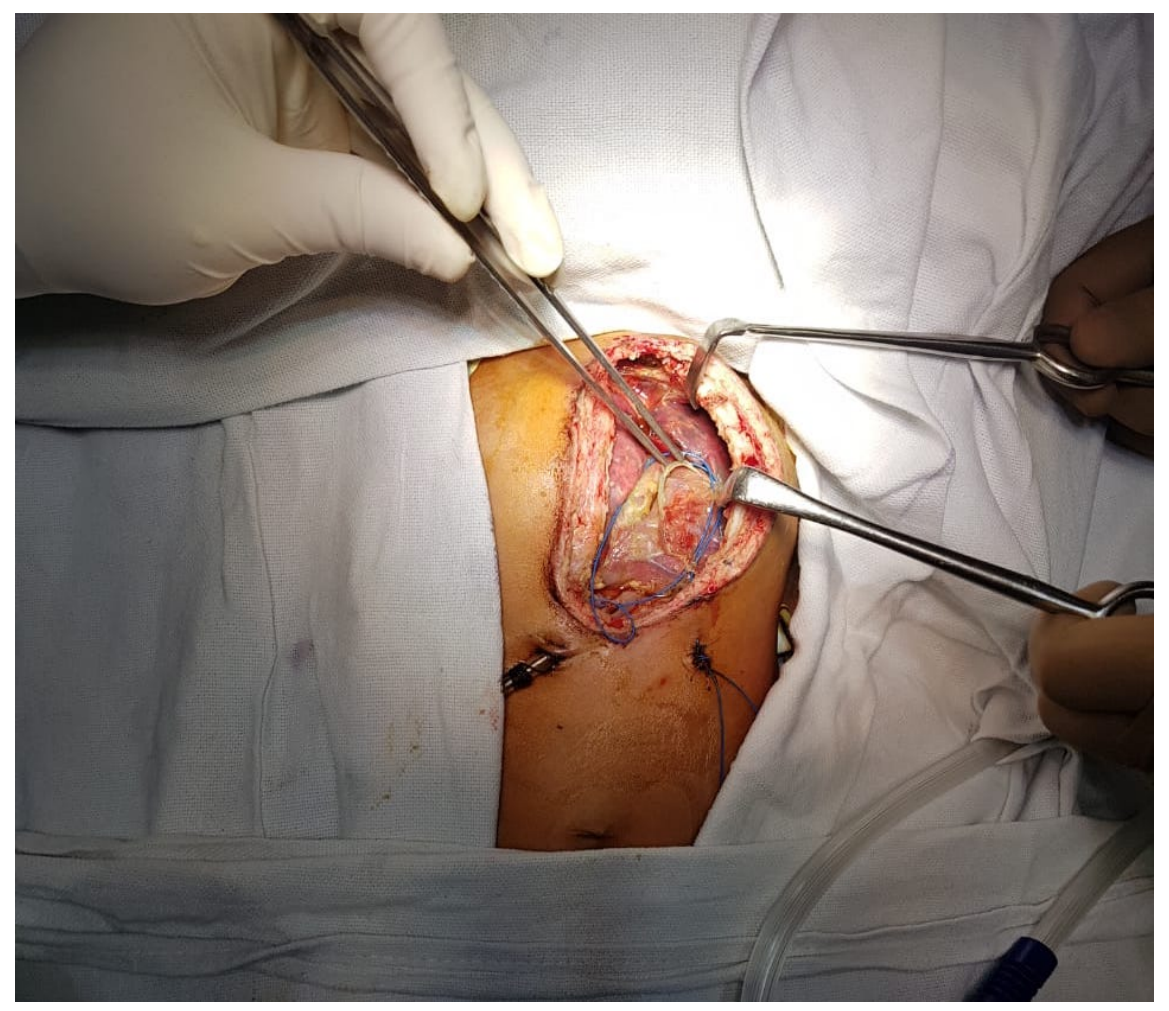

Figure-1-Surgical Site showing infected sternal and pericardial tissue 


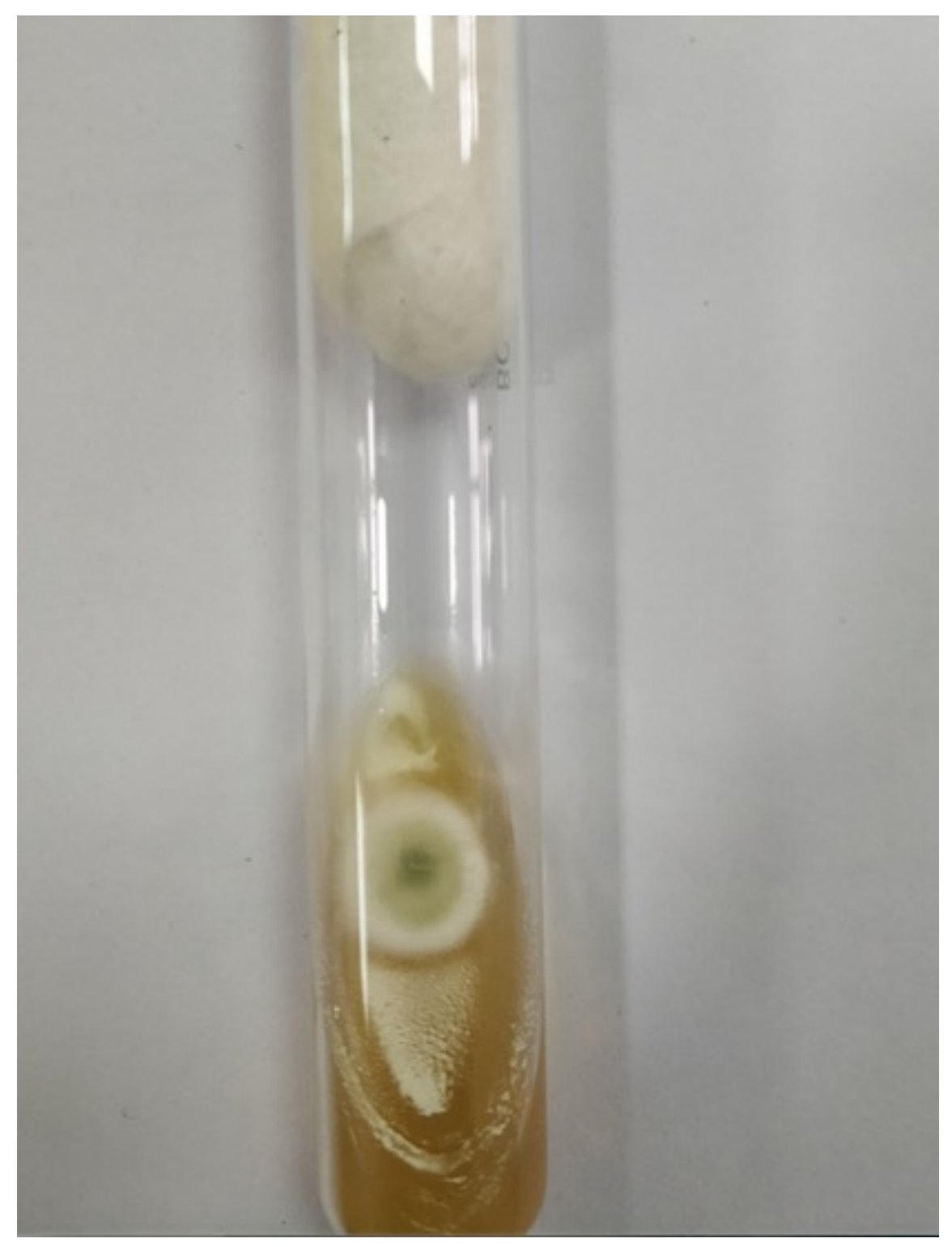

Figure-2- Sabouraud dextrose agar showing flat yellow-green colonies after 2-days of incubation at $37^{\circ} \mathrm{C}$ 


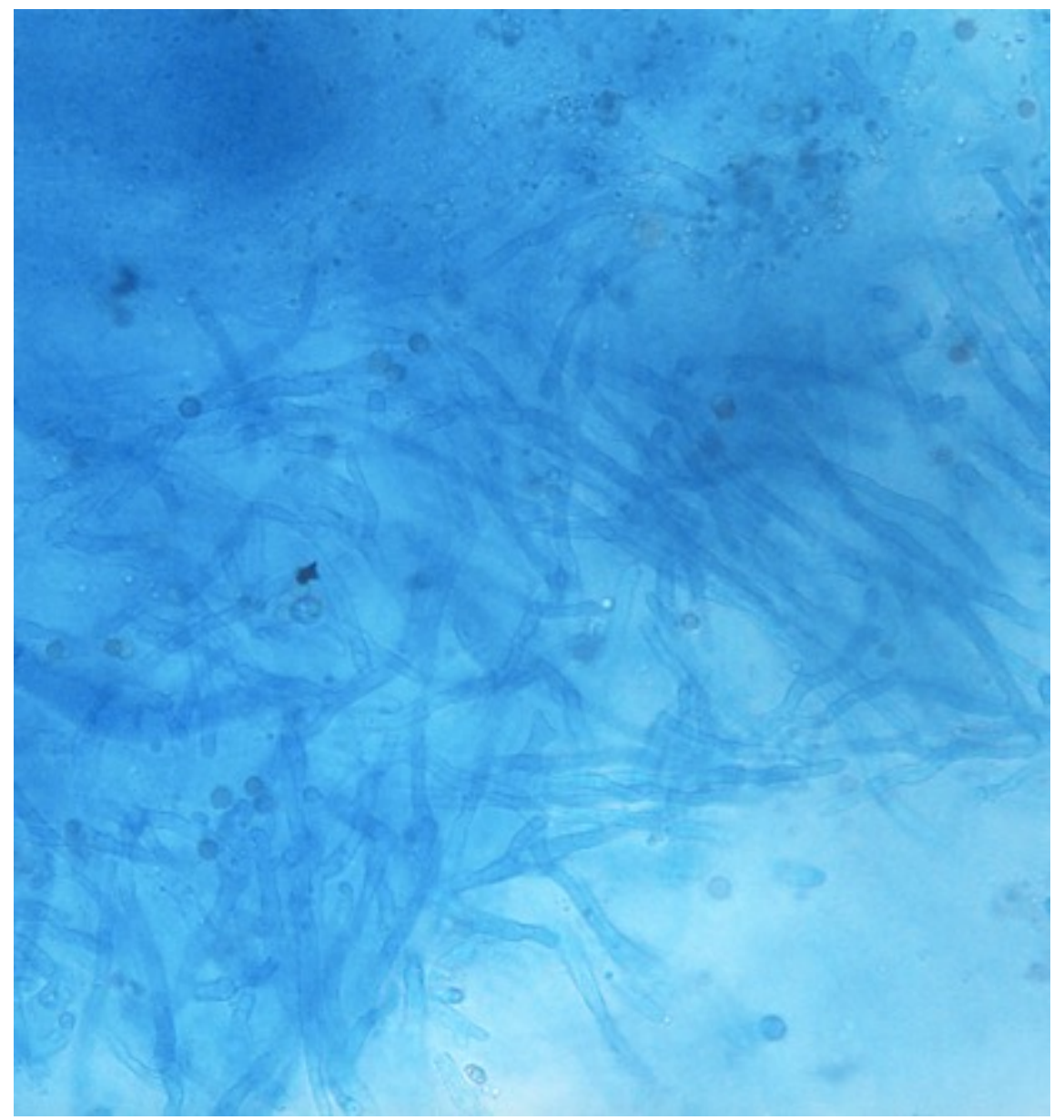

Figure-3- KOH mount showing hyaline, septate hyphae (40X) 


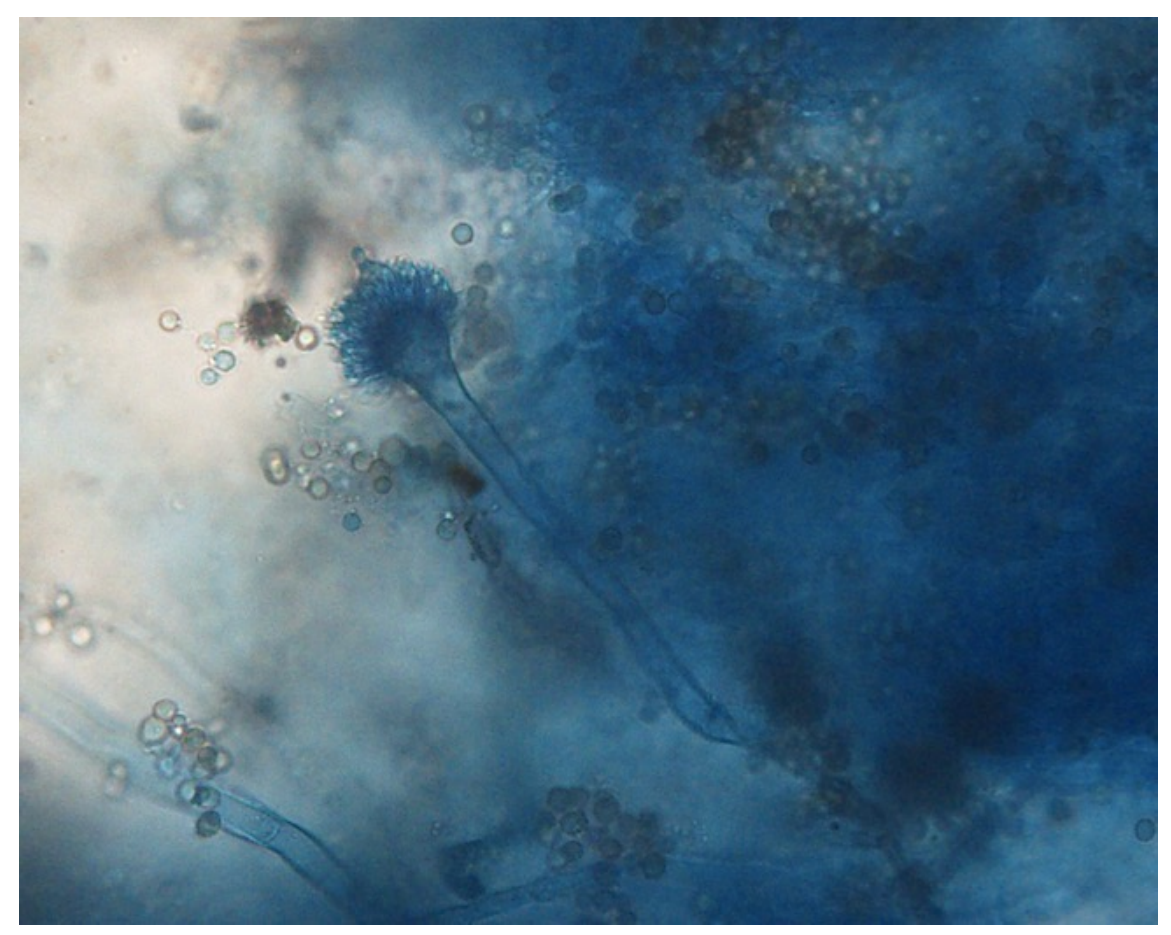

Figure-4- Lactophenol cotton blue preparation showing rough hyaline conidiophore with biseriate phialides, covering whole of the vesicle and globose, echinulate conidia, suggestive of Aspergillus flavus

\section{REFERENCES}

1. Pasqualotto AC, Denning DW: Post-operative aspergillosis. Clin Microbiol Infect. 2006, 12: 1060-1076

2. Kronman MP, Baden HP, Jeffries HE, Heath J, Cohen GA, Zerr DM. An investigation of Aspergillus cardiac surgical site infections in 3 paediatric patients. Am J Infect Control 2007; 35:332-7.

3. Vandecasteele SJ, Boelaert JR, Verrelst P, Graulus E, Gordts BZ. Diagnosis and treatment of Aspergillus flavus sternal wound infections after cardiac surgery. Clin Infect Dis 2002; 35:887-90. 